\title{
Andrea Claudio LEVI, a scientist and a friend
}

This collection of papers is dedicated to the scientific achievements of Andrea Claudio Levi on the occasion of his $72^{\text {nd }}$ birthday and consequent retirement from the University of Genoa, Italy.

Andrea was born in Milan in 1937, but spent most of his life in Genoa with few interruptions. He graduated in Physics in 1960 and began to teach and to research in the group of Prof. Giovanni Boato, involved at the time especially in the study of the separation factor of argon isotopes, giving theoretical support. He spent the year 1964 in Paris, doing theoretical research in plasma physics under the supervision of Prof. Theo Kahan: a short paper by Levi was presented to the Académie des Sciences by Prince Louis de Broglie (although Levi never had the opportunity to meet de Broglie...).

A little later, Levi left for Leiden, Holland: this period in Holland (1967-68) was very important, because of the strongly enriching interaction with Prof. Jan Beenakker.

Levi learned a considerable amount of molecular physics in Leiden, and had also e.g. the opportunity to explain a mysterious "General Motors" effect (so called because it had been found in a research laboratory of that company): a solid cylinder suspended in a molecular gas in a non-uniform temperature situation experiences an unexpected torque; Levi was able to show that the effect is due to the coupling between angular momentum and velocity of the molecules and related to the second derivatives of the temperature field.

Coming back to Genova, Andrea Levi was involved again with Prof. Boato, who was then starting to study atom-surface scattering. Levi studied the problem theoretically. This work lasted for a long time, and led to interesting results. One of the most well-known such result was a simple, but general calculation of diffraction intensities, including the solution of the rainbow puzzle.

At that time, many experimentalists measured the angular distribution of helium atoms back-reflected from a solid surface. Those who measured in a less refined fashion, with considerable noise, saw the scattering probability showing two characteristic "rainbow" maxima (so-called because of the analogy with optics). Those who measured

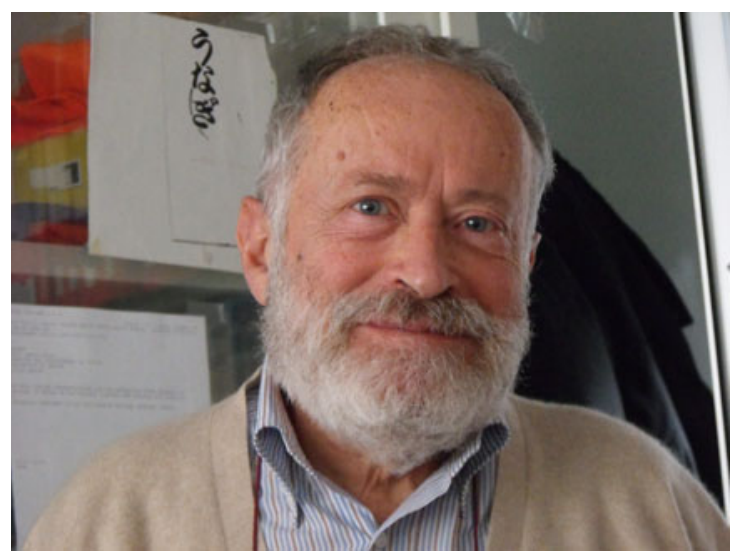

better, including Boato, saw beautiful diffraction peaks, but seemed to lose the rainbow. Levi explained that, quantum-mechanically, the rainbow structure is nothing but the envelope of the diffraction peaks; and, from this point of view, the rainbow was quite evident in Boato's data. Other contributions by Levi in that period (in the field of atom-surface scattering), equally important although less popular, referred to the Debye-Waller factor and to the inelastic scattering distribution.

Levi spent the year 1979 in Trento and the following year became full professor and was called to Naples, where he stayed during 1981. Then he came back to Genova and went on in his surface studies.

In 1987, and again for a longer period between 1989 and 1992 Levi was at the SISSA in Trieste: another very important experience, because of the intense collaboration with Erio Tosatti, one of the best Italian physicists. Levi continued to study surfaces, but this time under a much more general point of view. In particular, he studied surface phase transitions: roughening and surface melting. Both roughening and surface melting are extremely interesting subjects. Roughening can be studied in a more mathematically clean fashion, meeting fundamental methods of two-dimensional statistical mechanics. Levi, Tosatti and Trayanov proposed a new phenomenon: missing-row roughening, for the (110) surfaces of $\mathrm{Ni}, \mathrm{Cu}, \mathrm{Pd}, \mathrm{Ag}$. 
Subsequently this effect was experimentally found by several groups, including Bracco and Tatarek in Genova). Surface melting, on the other hand, affords an illuminating understanding of the solid-liquid interface: a difficult but fascinating matter.

Andrea Levi spent also some time in America (Waterloo, Ontario; San Diego, California; more recently, in 1998 , Princeton. New Jersey). He has studied in recent times structural and growth properties not only of surfaces, but also of clusters, which are fashionable and technologically important, and also instructive both for geometric and for chemical reasons. In particular, Levi studied the melting of $\mathrm{H}_{2}$ clusters (related to their possible superfluidity).

Ultimately, stimulated by new, fascinating experiments by the Cambridge group of William Allison on neon scattering from an adsorbate-covered surface, Levi has reconsidered his work of the Seventies (with Prof. Harry Suhl) on the Debye-Waller factor in slow-atom scattering from surfaces, discovering that it had a more fundamental meaning than previously suspected.

In the summer of 2009 the $26^{\text {th }}$ European Conference on Surface Science held in Parma saw the participation of many past co-workers of Prof. Levi who paid him a special tribute for his $72^{\text {nd }}$ birthday.

Andrea Levi has published 108 scientific articles, mostly in surface physics, but to many more he gave his contribution since talking with him about our preliminary results was always a pleasure and he very often had some important advice which ended up as an acknowledgment.

Andrea Levi is married with Maria Luisa Boero: they have a daughter, Valentina, living in England. Apart from physics, he has been active in the political life of Genoa and he should be remembered also for two books of collections of tales and essays, "L'acquisto della Terra" ("Buying the Earth", published with De Ferrari, Genoa) and "Il nome dell'Europa" ("The name of Europe" published with Giuntina, Florence).

Mario Agostino Rocca

Professor of Condensed Matter Physics Dipartimento di Fisica Università di Genova 